\title{
Long-term follow-up studies of Gamma Knife surgery with a low margin dose for vestibular schwannoma
}

\author{
Clinical article
}

\author{
*Shibin Sun, M.D., And Ali Liu, M.D. \\ Gamma Knife Center, Beijing Neurosurgical Institute, Tiantan Hospital, Capital University of Medical \\ Sciences, Beijing, China
}

\begin{abstract}
Object. The aim of this study was to assess long-term clinical outcomes in patients who underwent Gamma Knife surgery (GKS) with a low margin dose-14 Gy or less - to treat vestibular schwannoma (VS) unrelated to neurofibromatosis Type II.

Methods. Between December 1994 and December 2001, 200 patients with VSs underwent GKS, which was performed using the Leksell Gamma Knife model B. More than 10 years of follow-up is available in these patients. One hundred ninety patients (88 male and 102 female patients) were followed up using MRI (follow-up rate 95\%). The mean age of these patients was 50.6 years (range 10-77 years). Gamma Knife surgery was the primary treatment for VS in 134 cases $(70.5 \%)$ and was an adjunctive management approach in 56 cases $(29.5 \%)$. The median tumor margin dose was 13.0 Gy (range 6.0-14.4 Gy), and the median maximum tumor dose was 28.0 Gy (range 15.0-60.0 Gy). The median tumor volume was $3.6 \mathrm{~cm}^{3}\left(\right.$ range $\left.0.3-27.3 \mathrm{~cm}^{3}\right)$. The median duration of follow-up in these patients was 109 months (range 8-195 months).

Results. In the 190 patients, the latest follow-up MRI studies demonstrated tumor regression in 122 patients $(64.2 \%)$, stable tumor in 48 patients $(25.3 \%)$, and tumor enlargement in 20 patients $(10.5 \%)$. The total rate of tumor control was $89.5 \%$. Using the Kaplan-Meier method, the authors found the estimated 3-, 5-, 10-, and 15-year tumor control rates to be $95 \%, 93 \%, 86 \%$, and $79 \%$, respectively; and the estimated 3-, 5-, and 10-year hearing preservation rates to be $96 \%, 92 \%$, and $70 \%$, respectively. Twenty-six patients $(13.7 \%)$ exhibited transient mild facial palsy or facial spasm, and 2 patients $(1.1 \%)$ suffered persistent mild facial palsy. Thirty-nine patients $(20.5 \%)$ had transient trigeminal neuropathy, and 5 patients $(2.6 \%)$ suffered from persistent mild facial numbness. The incidence of persistent severe facial and trigeminal neuropathy was $0.0 \%$.

Conclusions. With a low prescribed margin dose of $14 \mathrm{~Gy}$ or less, GKS was confirmed to provide long-term tumor control for small to medium-sized VSs and largely to prevent cranial nerves from iatrogenic injury. Based on the findings of this study, GKS is also a reasonable option for the treatment of large, heterogeneously enhancing tumors without symptomatic brainstem compression. Gamma Knife surgery can preserve a high quality of life for most patients with VS who do not have symptomatic brainstem compression. Long-term follow-up is required because of the risk of delayed recurrence of VS.

(http://thejns.org/doi/abs/10.3171/2012.7.GKS12783)
\end{abstract}

\section{KeY Words - vestibular schwannoma - Gamma Knife surgery stereotactic radiosurgery - long-term follow-up}

$\mathrm{W}$ ELL-REGARDED articles about long-term outcomes in patients who have undergone GKS for VS have led to a growing acceptance of this approach as a safe and effective treatment modality for patients harboring these lesions. ${ }^{1-15}$ Due to an evolution in the prescribed margin dose in the early 1990s, the preservation rate of cranial nerve function has improved. Nowadays a relatively low dose of $12-13$ Gy directed to the tumor margin (to the $50 \%$ isodose surface) is generally accepted to be the most commonly used prescribed dose of GKS for VS. We have more than 15 years' ex-

Abbreviations used in this paper: GKS = Gamma Knife surgery; $\mathrm{TN}=$ trigeminal neuralgia; VS = vestibular schwannoma.

* Drs. Sun and Liu contributed equally to this work. perience with a low GKS prescribed margin dose of 14 Gy or less for VS, since the Gamma Knife Center at the Beijing Neurosurgical Institute opened in 1994. To assess the long-term clinical outcomes in patients who underwent GKS for VS with a margin dose of 14 Gy or less, we performed a retrospective study of our patients with VS who were treated between 1994 and 2001 and who had the potential for 10 or more years of follow-up.

\section{Methods}

\section{Patient Demographics}

Between December 1994 and December 2001, a consecutive series of 200 patients harboring VSs underwent 
GKS at the Gamma Knife Center of the Beijing Neurosurgical Institute. Adequate follow-up was available and included MRI examinations in 190 (95\%) of these patients. These 190 patients comprise the study group described in this article; the other 10 patients were lost to follow-up. Of these 190 patients, 88 (46.3\%) were male and $102(53.7 \%)$ were female. The mean age of these patients was 50.6 years (range 10-77 years). Gamma Knife surgery was performed as the primary treatment in 134 patients $(70.5 \%)$, in whom the diagnosis of VS was based on MRI findings alone. Gamma Knife surgery was the secondary treatment, following microsurgery, in 56 patients $(29.5 \%)$ in whom the diagnosis was histopathologically confirmed. Patients with neurofibromatosis Type II were excluded from this study.

\section{Clinical Features}

The median tumor volume was $3.6 \mathrm{~cm}^{3}$ (range $0.3-$ $\left.27.3 \mathrm{~cm}^{3}\right)$. Classified by the tumor's imaging appearance, 118 patients $(62.1 \%)$ harbored homogeneously enhancing (purely solid) tumors, and 72 patients $(37.9 \%)$ harbored heterogeneously enhancing (mixed cystic and solid) tumors. Hearing and facial symptoms were assessed at baseline (pre-GKS) and during follow-up according to the Gardner-Robertson and House-Brackmann grading systems, respectively. Pre-GKS and post-GKS hearing and facial function status are shown in Table 1. Tinnitus was reported by 124 patients (65.3\%). At the time of GKS, 137 patients $(72.1 \%)$ had normal facial function.

\section{Radiosurgical Technique}

In all these patients, the Leksell model G stereotactic frame (Elekta Instruments) was applied following administration of a local anesthetic agent and a mild oral sedative. High-resolution contrast-enhanced MRI was performed for tumor localization and for follow-up examinations. During the period in which this cohort of patients was treated, the Leksell Gamma Knife model B was used at our center. Elekta proprietary radiosurgery treatment planning software was used for all cases: the KULA system was used before May 1996 and the GammaPlan thereafter. The median tumor margin dose was 13.0 Gy (range 6.0-14.4 Gy), and the median maximum tumor dose was $28.0 \mathrm{~Gy}$ (range 15.0-60.0 Gy). The median prescribed isodose line was $45.0 \%$ (range $20 \%-68 \%$ ), and the median number of isocenters was 4 (range 2-9). Because of a large tumor volume or another reason, 10 patients in this group received a margin dose lower than 12 Gy.

\section{Follow-Up Evaluation}

After GKS, MRI studies were periodically obtained in all 190 patients in the study group. For the purpose of this article, the criteria used to evaluate the tumor response on MR images after GKS are classified into three categories: 1) tumor regression is defined as a post-GKS tumor volume that is $10 \%$ less than the pre-GKS tumor volume or $\mathrm{a} \geq 2 \mathrm{~mm}$ shrinkage of the lesion along one axis; 2) stable tumor is defined as a post-GKS tumor volume change that is within $10 \%$ or $<2 \mathrm{~mm}$ change in the lesion along one axis; and 3) tumor enlargement is defined as a post-GKS tumor volume that is $10 \%$ greater than the pre-GKS tumor volume or a $\geq 2 \mathrm{~mm}$ growth in the lesion along one axis.

\section{Statistical Analysis}

The Kaplan-Meier product-limit method was used to calculate estimated rates of tumor control and hearing function preservation. An analysis of the correlation of potential prognostic factors with tumor control was performed using the following groups: patient sex (male vs female); patient age ( $<65$ years vs $\geq 65$ years); margin dose ( $<13$ Gy vs $\geq 13$ Gy); prior surgery (no vs yes); tumor volume $\left(<5 \mathrm{~cm}^{3}\right.$ vs $5-10 \mathrm{~cm}^{3}$ vs $\left.\geq 10 \mathrm{~cm}^{3}\right)$; and imaging appearance (homogeneously enhancing vs heterogeneously enhancing). A univariate analysis was performed on Kaplan-Meier plots by using the log-rank statistic, with a $p$ value of $<0.05$ considered significant. A multivariate analysis was performed using the Cox regression model $(95 \% \mathrm{CI})$. We used standard statistical processing software (SPSS version 17.0; SPSS, Inc.) for the analyses.

\section{Results}

\section{Tumor Control}

The median duration of follow-up was 109 months (range 8-195 months). The duration of follow-up was $\geq$ 60 months in 132 patients $(69.5 \%)$ and $\geq 120$ months in 86 patients $(45.3 \%)$. Of the 190 patients, the latest follow-up MRI studies demonstrated tumor regression in 122 patients $(64.2 \%)$, stable tumor in 48 patients $(25.3 \%)$, and tumor enlargement in 20 patients $(10.5 \%)$. The total rate of tumor control was $89.5 \%$. We observed a post-GKS loss of enhancement in 134 patients (70.5\%) in this group. Of

TABLE 1: Hearing status and facial nerve function in 190 patients who underwent GKS for VSs

\begin{tabular}{ccccccc}
\hline Gardner-Robertson & \multicolumn{2}{c}{ No. of Cases (\%) } & House-Brackmann Facial & \multicolumn{2}{c}{ No. of Cases (\%) } \\
\cline { 2 - 3 } \cline { 6 - 7 } Hearing Scale Grade & Pre-GKS & Post-GKS & Nerve Function Grade & Pre-GKS & Post-GKS \\
\hline I & $3(1.6)$ & $3(1.6)$ & I & II & $137(72.1)$ & $122(64.2)$ \\
II & $19(10)$ & $15(7.9)$ & & III & $4(2.1)$ & $17(8.9)$ \\
III & $83(43.7)$ & $60(31.6)$ & IV & $20(10.5)$ & $15(7.9)$ \\
IV & $52(27.4)$ & $67(35.3)$ & V & $11(5.8)$ & $11(5.8)$ \\
V & $33(17.4)$ & $45(23.7)$ & & VI & $5(2.6)$ & $4(2.1)$ \\
& & & & $190(100)$ & $190(100)$ \\
\hline
\end{tabular}


the 20 patients in whom tumor enlargement was shown on the latest MR images, 7 underwent repeated GKS, 7 underwent microsurgery, 2 received ventriculoperitoneal shunts, and 4 were kept under close observation. Sixteen patients in our cohort died during the follow-up period. Twelve patients died of causes unrelated to the VS, with maintenance of a high quality of life following GKS, and 4 patients died of VS progression. The Kaplan-Meier-estimated 3-, 5-, 10-, and 15-year tumor control rates were 95\%, 93\%, 86\%, and 79\%, respectively (Fig. 1).

\section{Cranial Nerve Function}

The post-GKS hearing and facial function statuses in the patients are shown in Table 1; all data were collected after the first radiosurgery. After GKS, serviceable hearing (Gardner-Robertson Grades I and II) was detected in 18 patients $(9.5 \%)$, hearing loss of some degree (Gardner-Robertson Grades III and IV) was detected in 127 patients (66.8\%), and complete hearing loss (GardnerRobertson Grade V) was detected in 45 patients (23.7\%). Tinnitus still existed in 69 patients, although the overall rate of improvement in tinnitus was $44.4 \%$.

We observed improvement in hearing in 12 patients (6.3\%), stabilization of hearing in 131 patients (68.9\%), and deterioration of hearing function in 47 patients (24.7\%). The Kaplan-Meier-estimated 3-, 5-, and 10-year hearing preservation rates were $96 \%, 92 \%$, and $70 \%$, respectively (Fig. 2).

In our cohort of patients, 26 patients (13.7\%) exhibited transient mild facial palsy or facial spasm, and 2 patients $(1.1 \%)$ suffered from persistent mild facial palsy. Thirty-nine patients $(20.5 \%)$ had transient trigeminal neuropathy, and 5 patients (2.6\%) suffered from persistent mild facial numbness. The incidence of persistent severe facial and trigeminal neuropathy was $0.0 \%$. Among 10 patients who presented with secondary TN, 8 patients experienced improvement (to varying degrees) due to tumor regression after GKS.

\section{Salvage Treatment}

Eighteen patients (9.5\%) underwent repeated GKS, 6

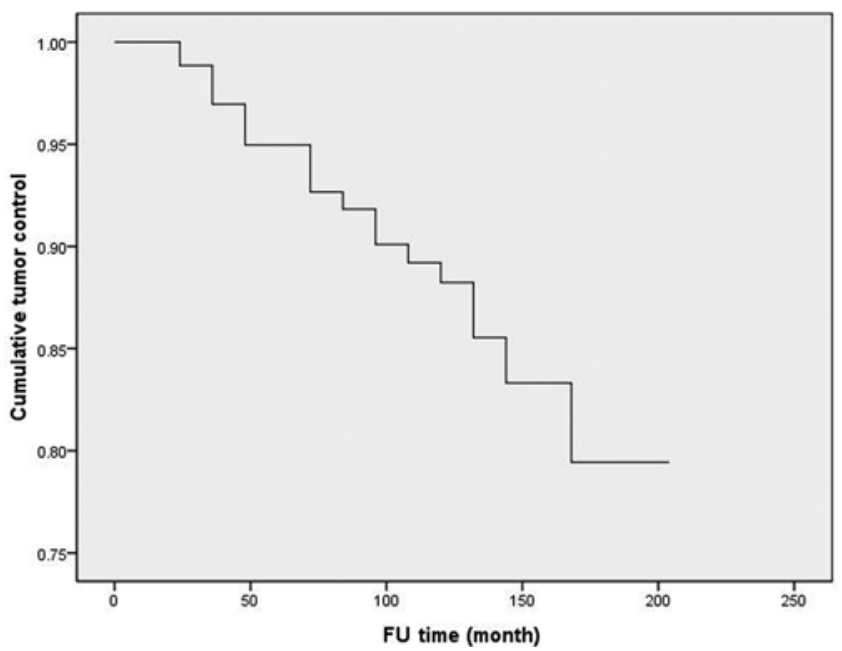

FIG. 1. Graph depicting a Kaplan-Meier curve of cumulative tumor control in 190 patients who underwent GKS for VSs. FU = follow-up.

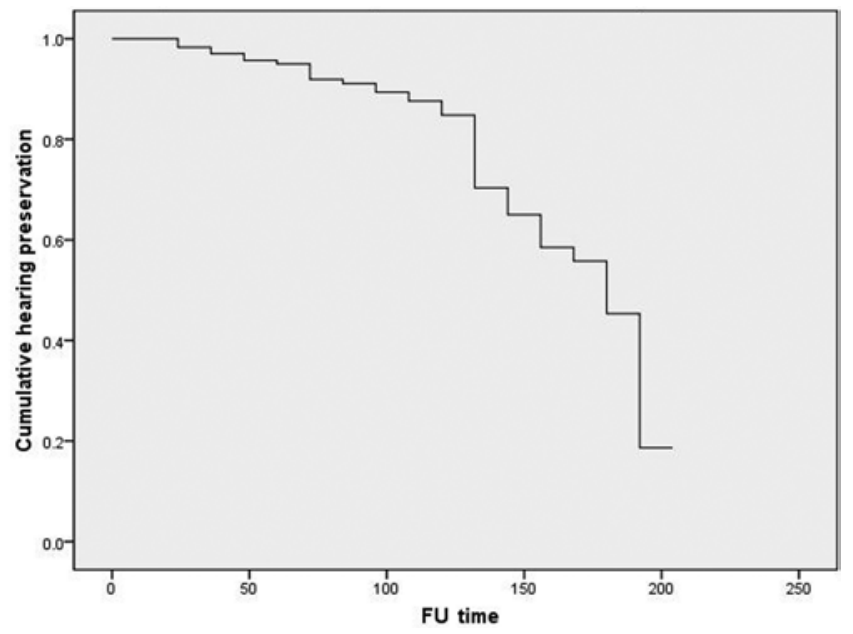

FIG. 2. Graph showing a Kaplan-Meier curve of cumulative hearing preservation in 190 patients who underwent GKS for VSs.

patients $(3.2 \%)$ required placement of a ventriculoperitoneal shunt due to hydrocephalus, 10 patients $(5.3 \%)$ underwent microsurgical resection, and 156 patients $(82.1 \%)$ required no additional intervention during the follow-up period after GKS.

In the group of 18 patients who were treated with additional GKS, 8 patients underwent planned repeated GKS due to the large volume of their VSs, 7 patients underwent a second GKS due to an enlargement of tumor volume after the initial GKS, and 3 patients required repeated GKS due to development of secondary TN. Pain relief was achieved in all patients after repeated GKS. The mean tumor volume in this group was $7.1 \mathrm{~cm}^{3}$ (range $0.3-18.7 \mathrm{~cm}^{3}$ ). In this group of 18 patients, the mean margin dose at the first GKS was 12.2 Gy (range 9-14 Gy), and the mean margin dose at the second GKS was 11.1 Gy (range 10-12 Gy; cases with TN were excluded). The mean interval between the first and second GKSs was 56.8 months (range 12-168 months). In this cohort of patients, the rate of tumor control was $88.9 \%$ (16 of 18 patients), the incidence of persistent mild facial neuropathy was $11.1 \%$ (2 of 18 patients), and the incidence of persistent mild trigeminal neuropathy was $22.2 \%$ (4 of 18 patients).

\section{Discussion}

\section{Long-Term Results of Tumor Control After GKS}

It has been more than 40 years since the first GKS for VS was reported in 1971, and increasing numbers of clinicians have become interested in long-term results $(>10$ years) following GKS for VS. Recently reported long-term outcomes are listed in Table 2., $, 8,15$ The Kaplan-Meier-estimated 5-year and 10-year tumor control rates in our patients were $93 \%$ and $86 \%$, which are similar to the longterm results reported by Chung and colleagues ${ }^{1}(95 \%$ at 7 years) and Hasegawa et al. ${ }^{5}$ (87\% at 10 years). The reason that the tumor control rates in the current study and those reported by Hasegewa et al. are slightly less than those in other reports may be because the investigators treated much larger volumes and had much longer follow-up times. 
TABLE 2: Comparison of long-term results at Beijing Neurosurgical Institute with other Gamma Knife centers*

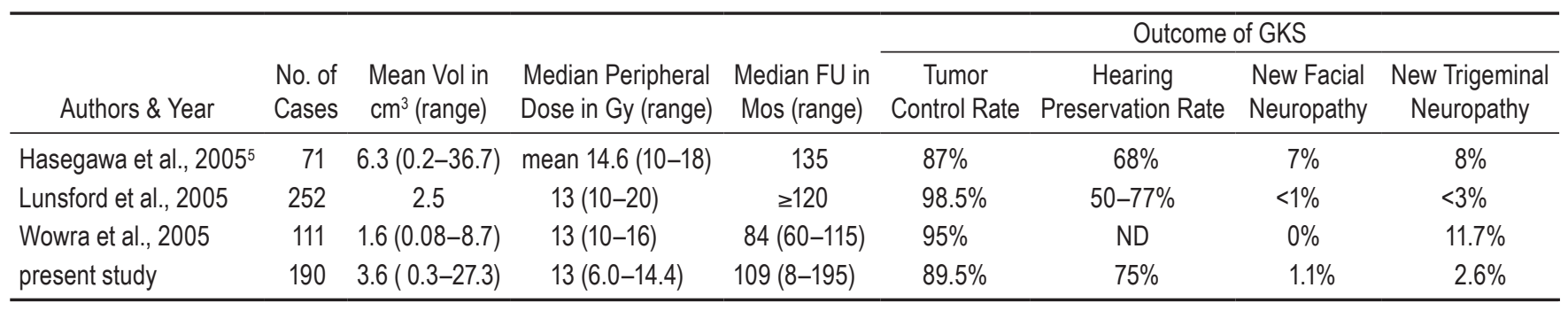

* FU = Follow-Up; ND = not determined.

There has been a reduction in the prescribed peripheral dose from 16-20 Gy to 12-14 Gy in most Gamma Knife centers whose results have been reported, $, 1,5,8,9,13,15$ but no difference in tumor control rates has been associated with this decrease, and there is also no evident difference in tumor control rates between these reports and the current study (prescribed peripheral dose 12-14 Gy). Thus there is no doubt that GKS could provide long-term, even lifelong tumor growth arrest for VS in most patients who receive a 12-14 Gy prescribed margin dose (Fig. 3).

For those patients who underwent salvage GKS for progressive VSs, the rate of tumor control after retreatment with GKS was $88.9 \%$, resembling the results reported by Dewan and Norén ${ }^{3}$ (an $82 \%$ tumor control rate). On the basis of these study findings, it can be concluded that retreatment with GKS should be considered an optional management strategy for recurrent VSs with small to medium-sized volumes.

The mean documented tumor growth time after GKS in this patient group was 53 months (range 12-168 months). Tumor enlargement was revealed in 5 patients by follow-up MR imaging studies conducted longer than 5 years after GKS (Fig. 4). Our results seem to be at variance with the view that GKS remains effective for more than 5 years, and we feel that a 5-year follow-up after GKS for VS is not sufficient to determine the ultimate extent of tumor control. We believe that long-term, even lifelong follow-up is essential for VS after GKS.

\section{Factors Associated With Tumor Control}

Patient sex $(\mathrm{p}=0.158, \mathrm{HR}=1.623,95 \%$ CI $0.828-$ $3.180)$, patient age ( $\mathrm{p}=0.486, \mathrm{HR}=1.325,95 \%$ CI $0.601-$ $2.921)$, margin dose $(\mathrm{p}=0.002, \mathrm{HR}=0.526,95 \% \mathrm{CI}$ $0.348-0.797)$, prior surgery $(\mathrm{p}=0.923, \mathrm{HR}=0.965,95 \%$ CI 0.465-2.003), tumor volume $(\mathrm{p}=0.000, \mathrm{HR}=2.097$, $95 \%$ CI 1.420-3.098), and imaging appearance $(\mathrm{p}=0.665$, $\mathrm{HR}=1.158,95 \%$ CI 0.597-2.246) were included in the univariate analysis of tumor control. Margin dose and tumor volume affected tumor control significantly in the univariate analysis. In the multivariate analysis, in which patient sex $(p=0.248)$, patient age $(p=0.862)$, margin dose $(p=$ $0.241)$, prior surgery $(\mathrm{p}=0.891)$, tumor volume $(\mathrm{p}=0.000$, $\mathrm{HR}=2.097,95 \%$ CI 1.420-3.098), and imaging appearance $(p=0.609)$ were included, tumor volume was the only factor that affected tumor control significantly. Similarly Hasegawa et al. ${ }^{5}$ reported that tumor volume remained a significant prognostic factor for tumor control in both univariate and multivariate analyses.
From the illustrative cases of treatment failure reported in this article, we noted that for large VSs with symptomatic brainstem compression, performing GKS led to serious consequences. Tumor volume is the dominant element affecting tumor control: the larger the tumor volume, the lower the tumor control rate. The worst prognostic factors were found to be present in patients harboring a large tumor concomitant with symptomatic brainstem compression.

\section{Gamma Knife Surgery for Large VSs}

Gamma Knife surgery alone has become the standard treatment for small to medium-sized VSs because it results in long-term tumor control with low incidences of morbidity and mortality. Nevertheless, there is ongoing
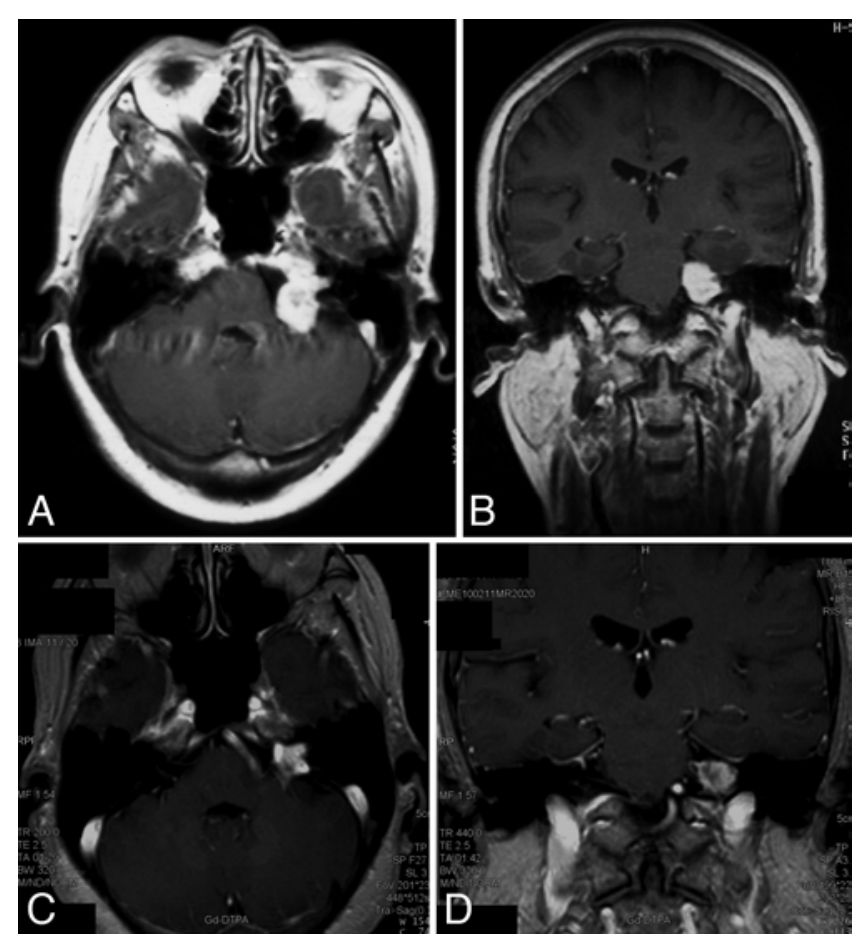

FIG. 3. Magnetic resonance images depicting a $5.3-\mathrm{cm}^{3}$ homogeneously enhancing VS obtained in a 59 -year-old woman. The lesion was treated with a 12.8-Gy margin dose at the $40 \%$ isodose line. A and B: Axial (A) and coronal (B) images obtained before GKS. C and D: Axial (C) and coronal (D) images demonstrating tumor regression 180 months after GKS. 

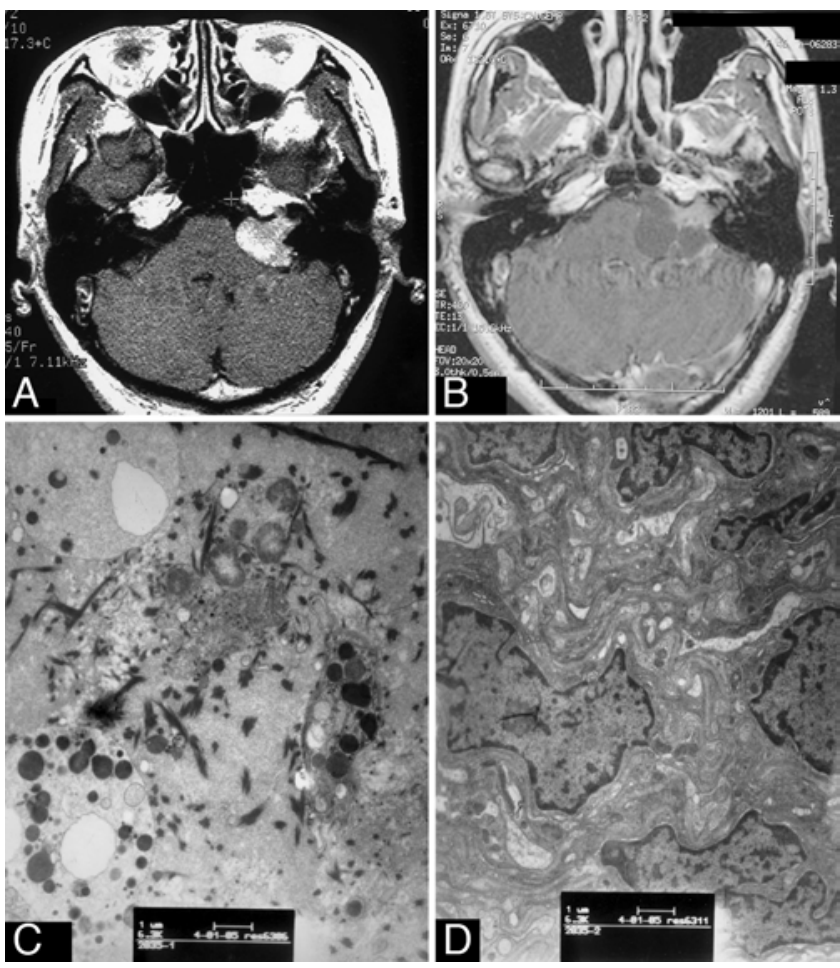

FIG. 4. A: Axial MR image obtained at the time of GKS, showing a nearly $10-\mathrm{cm}^{3}$ homogeneously enhancing VS obtained in a 39 -year-old woman. The lesion was treated with a $12-G y$ margin dose at the $45 \%$ isodose line. B: Follow-up axial MR image obtained 96 months after GKS, revealing a delayed enlarging cystic change and compression of the brainstem by the tumor. The patient underwent craniotomy. $\quad \mathbf{C}$ : Electron micrograph showing massive exudation of fibrin and formation of collagenous tissue in the lesion. D: Electron micrograph demonstrating an Antoni B-type schwannoma cell with an irregular nucleus and reticular cytoplasm. Original magnifications $\times 6300$.

contention about the efficacy of GKS for large VSs (generally thought of as tumors $>10 \mathrm{~cm}^{3}$ ). The lack of reliable data has hindered neurosurgeons from making recommendations for either microsurgery or GKS in patients who are poor operative risks and harbor large-volume VSs. Chung and coworkers ${ }^{2}$ in 2010 reported 21 cases of large-volume VSs treated by GKS. Those authors also reviewed other papers and summarized good tumor control rates (84\%-100\%), excellent preservation of cranial nerve function, and low treatment failure rates $(0 \%-12 \%)$ with the use of GKS for large-volume VSs. In the series reported by Chung and coworkers ${ }^{2}$ the mean tumor volume was $17.3 \mathrm{~cm}^{3}$, the margin dose was 10-14 Gy, the mean follow-up time was 5.5 years, and the tumor control rate was $90.5 \%$ with a $100 \%$ facial nerve preservation rate. In this report, only 5 patients required surgical intervention after GKS.

In our group, 28 patients harbored VSs with tumor volumes $>10 \mathrm{~cm}^{3}$. For these patients, the mean tumor volume was $14.3 \mathrm{~cm}^{3}$, the margin dose was $6-12 \mathrm{~Gy}$, the mean follow-up time was 6.2 years, and the tumor control rate was $78.6 \%$, which we acknowledge is far less than usually reported. Of the 20 patients in whom GKS did not control the VS, 11 patients $(55 \%)$ harbored $\geq 10 \mathrm{~cm}^{3}$ tumors, and 9 patients $(45 \%)$ had severe symptomatic brainstem compression. We conclude that, in our experience, GKS was absolutely not suitable for VSs $\geq 10 \mathrm{~cm}^{3}$ with concomitant symptomatic brainstem compression, especially when the tumor was found to be homogeneously enhancing on MRI studies. Although the statistical data showed no remarkable difference between homogeneously enhancing tumors and heterogeneously enhancing tumors, some cases revealed that heterogeneously enhancing tumors were more likely to manifest distinct volume changes (Fig. 5). On the basis of the cases successfully managed at our center, we conclude that single GKS or staged GKS is suitable for large-volume $(\geq 10$ $\mathrm{cm}^{3}$ ) heterogeneously enhancing VSs without symptomatic brainstem compression. Appropriate decreases in the prescribed margin dose could contribute to a reduction in complications from GKS.

\section{Long-Term Results of Cranial Nerve Function After GKS}

With the development and increasing sophistication of microsurgical and radiosurgical techniques, there is a tendency for the goals of VS management to emphasize the preservation of cranial nerve function and the maintenance of a high quality of life as emphatically as tumor control. With the reduction in the prescribed peripheral dose from 16-20 Gy to 12-14 Gy at most Gamma Knife centers, ${ }^{1,5,8-10,15}$ there has been a falloff in the rate of cranial nerve morbidity. Many groups have reported in the
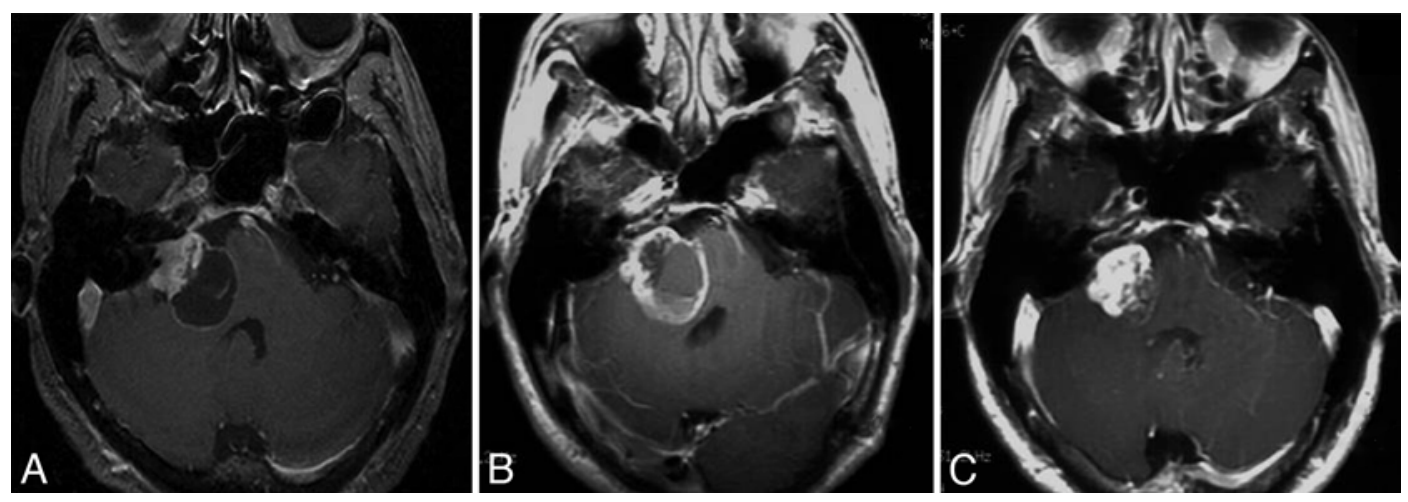

FIG. 5. Axial MR images obtained in a 65 -year-old man who presented with a $>10-\mathrm{cm}^{3}$ heterogeneously enhancing tumor. The lesion was treated with a $13-G y$ margin dose at the $45 \%$ isodose line. The serial images document the process of dramatic tumor shrinkage: at the time of GKS (A); 12 months after GKS (B); and 25 months after GKS (C). 
literature ${ }^{1-15}$ that between $52 \%$ and $83.4 \%$ of patients with useful pre-GKS hearing function have preserved useful hearing after GKS for VS. The rate of hearing function preservation in our series was $75.3 \%$, which is well within this range. Tinnitus still existed in more than half of this group. Norén ${ }^{9}$ has noted that tinnitus is rarely changed by the treatment.

Based on our experience, we are in agreement with two viewpoints from other reports: the larger the tumor volume, the lower the hearing preservation rate; ${ }^{13}$ and the longer the follow-up time, the lower the hearing preservation rate. ${ }^{11}$ The Kaplan-Meier-estimated hearing preservation rate in our experience showed a remarkable declining trend during the follow-up period. The guidelines from the International RadioSurgery Association conclude that facial and trigeminal nerve function can now be preserved in the majority of patients $(>95 \%)$ when the previously used tumor margin dose of $18-20$ Gy is reduced to 12-14 Gy. The published results from Hasegawa et al., ${ }^{5}$ Chung and colleagues, ${ }^{1}$ and Wowra et al., ${ }^{15}$ and those from the current study also support this statement. It is worth noting that the incidence of persistent mild facial and trigeminal neuropathy was 33.3\% in VS cases managed with repeated GKS, which was a higher rate than that observed for patients treated with a single GKS in the current study. We believe that the incidence of facial and trigeminal function injury will be raised after repeated GKS.

\section{Conclusions}

With a $12-14$ Gy prescribed margin dose, GKS is confirmed to provide long-term tumor control for small to medium-sized VS and largely to prevent cranial nerves from iatrogenic injury. In addition, based on our results, GKS is a reasonable treatment option for large, heterogeneously enhancing tumors without symptomatic brainstem compression. Gamma Knife surgery can preserve a high quality of life for most patients with VS without symptomatic brainstem compression. Long-term follow-up is required because of the risk of delayed recurrence of VS.

\section{Disclosure}

The authors report no conflict of interest concerning the materials or methods used in this study or the findings specified in this paper.

Author contributions to the study and manuscript preparation include the following. Conception and design: both authors. Acquisition of data: both authors. Analysis and interpretation of data: Sun. Drafting the article: Sun. Critically revising the article: both authors. Reviewed submitted version of manuscript: both authors. Approved the final version of the manuscript on behalf of both authors: Sun. Statistical analysis: Sun. Study supervision: Liu.

\section{Acknowledgment}

The authors thank Jonathan Knisely, M.D., Chief, Division of Radiosurgery and Stereotactic Program and Co-Director for Radiosurgery and Stereotactic Radiation Therapy in the Department of
Radiation Medicine at the North Shore-Long Island Jewish Health System, for his kind assistance in reviewing this article.

\section{References}

1. Chung WY, Liu KD, Shiau CY, Wu HM, Wang LW, Guo WY, et al: Gamma knife surgery for vestibular schwannoma: 10year experience of 195 cases. J Neurosurg 102 Suppl:87-96, 2005

2. Chung WY, Pan DH, Lee CC, Wu HM, Liu KD, Yen YS, et al: Large vestibular schwannomas treated by Gamma Knife surgery: long-term outcomes. Clinical article. J Neurosurg 113 Suppl:112-121, 2010

3. Dewan S, Norén G: Retreatment of vestibular schwannomas with Gamma Knife surgery. J Neurosurg 109 Suppl:144-148, 2008

4. Hasegawa T, Fujitani S, Katsumata S, Kida Y, Yoshimoto M, Koike J: Stereotactic radiosurgery for vestibular schwannomas: analysis of 317 patients followed more than 5 years. Neurosurgery 57:257-265, 2005

5. Hasegawa T, Kida Y, Kobayashi T, Yoshimoto M, Mori Y, Yoshida J: Long-term outcomes in patients with vestibular schwannomas treated using gamma knife surgery: 10-year follow up. J Neurosurg 102:10-16, 2005

6. Inoue HK: Low-dose radiosurgery for large vestibular schwannomas: long-term results of functional preservation. J Neurosurg 102 Suppl:111-113, 2005

7. Iwai Y, Yamanaka K, Shiotani M, Uyama T: Radiosurgery for acoustic neuromas: results of low-dose treatment. Neurosurgery 53:282-288, 2003

8. Lunsford LD, Niranjan A, Flickinger JC, Maitz A, Kondziolka D: Radiosurgery of vestibular schwannomas: summary of experience in 829 cases. J Neurosurg 102 Suppl:195-199, 2005

9. Norén G: Long-term complications following gamma knife radiosurgery of vestibular schwannomas. Stereotact Funct Neurosurg 70 (Suppl 1):65-73, 1998

10. Norén G, Greitz D, Hirsch A, Lax I: Gamma knife surgery in acoustic tumours. Acta Neurochir Suppl (Wien) 58:104107,1994

11. Ogunrinde OK, Lunsford LD, Flickinger JC, Kondziolka DS: Cranial nerve preservation after stereotactic radiosurgery for small acoustic tumors. Arch Neurol 52:73-79, 1995

12. Paek SH, Chung HT, Jeong SS, Park CK, Kim CY, Kim JE, et al: Hearing preservation after gamma knife stereotactic radiosurgery of vestibular schwannoma. Cancer 104:580-590, 2005

13. Prasad D, Steiner M, Steiner L: Gamma surgery for vestibular schwannoma. J Neurosurg 92:745-759, 2000

14. van Eck AT, Horstmann GA: Increased preservation of functional hearing after gamma knife surgery for vestibular schwannoma. J Neurosurg 102 Suppl:204-206, 2005

15. Wowra B, Muacevic A, Jess-Hempen A, Hempel JM, MüllerSchunk S, Tonn JC: Outpatient gamma knife surgery for vestibular schwannoma: definition of the therapeutic profile based on a 10-year experience. J Neurosurg 102 Suppl:114-118, 2005

Manuscript submitted April 20, 2012.

Accepted July 24, 2012.

Please include this information when citing this paper: DOI: 10.3171/2012.7.GKS12783.

Address correspondence to: Shibin Sun, M.D., Gamma Knife Center, Beijing Neurosurgical Institute, Tiantan Hospital, Capital University of Medical Sciences, No. 6 Chong wen qu Tiantanxili, Beijing, China 100050. email: ssbwyl@vip.sina.com. 